\title{
Addison's disease in a patient with a history of Hashimoto's thyroiditis (Schmidt's syndrome) and idiopathic thrombocytopenic purpura: Report of case and review of literature
}

\author{
B.W. MEEKER, D.O. \\ Milwaukee, Wisconsin \\ J.D. KING, D.O. \\ D.A. ARNOLD, D.O. \\ Davenport, Iowa
}

Deficiencies in cortisol and aldosterone result in the characteristic clinical manifestations of Addison's disease. When the disease presents as a life-threatening emergency, treatment consists of rapid rehydration and replacement of these steroids. The most common cause is "idiopathic atrophy," which is thought to be autoimmune destruction. It is now recognized that certain autoimmune diseases correlate with specific HLA haplotypes. The association of autoimmune Addison's disease and autoimmune hypothyroidism has been labeled "Schmidt's syndrome," which was present in the case reported. This patient also had a history of idiopathic thrombocytopenic purpura; this too is associated with HLA-B8. Patients who have an autoimmune disease related to a specific HLA haplotype seem to be at higher risk for developing other diseases associated with the same HLA antigens. HLA typing is still expensive and generally unavailable, but this situation may improve with developing technology. When an autoimmune disease is diagnosed, it is prudent to have a high index of suspicion for related autoimmune processes.

Whatever the cause, Addison's disease is destruction of the adrenal cortex. The resulting primary deficiencies of cortisol and aldosterone account for the clinical manifestations and result in compensatory increases in the production of adrenocorticotropin hormone (ACTH) and renin.

Lack of aldosterone results in impaired ability to conserve sodium and excrete potassium. The excessive excretion of sodium causes decreased extracellular fluid volume, with weight loss, hypovolemia, hypotension, weakness, postural syncope, and shock. Also manifested are decreased cardiac output, decreased renal blood flow, and increased renin production. The chest $\mathrm{x}$-ray may demonstrate decreased cardiac size, and laboratory evaluation will reveal prerenal azotemia. The impaired renal secretion of potassium and hydrogen ions will result in hyperkalemia, mild acidosis, and, if severe, cardiac asystole.

Manifestations of cortisol deficiency include nausea, vomiting, abdominal pain, anorexia, and weight loss. The patient also will exhibit apathy, weakness, wasting of fat deposits, and fasting hypoglycemia. The impaired ability to excrete free water couples with the aldosterone effect to produce hyponatremia and hypotension. The patient will have an impaired tolerance to stress, with all of the symptoms becoming more pronounced during trauma, infection, or fasting. The compensatory increase of ACTH and other melanocyte-stimulating hormones will result in mucocutaneous hyperpigmentation.

The most common etiology of Addison's disease is "idiopathic atrophy," which is now believed to be autoimmune destruction. It is common to see concomitant autoimmune diseases of other tissues leading to hypothyroidism, hypoparathyroidism, diabetes mellitus, primary ovarian failure, or pernicious anemia. Less common causes include tuber- 
culosis (formerly the principal cause), systemic fungal infections, metastatic carcinoma, and amyloidosis. A rare cause is adrenal apoplexy from fulminant meningococcemia (Waterhouse-Friderichsen syndrome).

Onset is insidious, and the compensatory increases in ACTH and renin can maintain homeostasis until 90 percent of the adrenal cortex is destroyed. However, with limited adrenal reserve, the disease will manifest during a period of stress, such as infection, trauma, or surgery.

Addisonian crisis is a life-threatening medical emergency; it calls for rapid intravenous infusion of physiologic saline solution, because as much as 20 percent of the extracellular fluid volume may be depleted. The diagnosis is clinical, and protocols have been developed to diagnose and treat Addison's disease simultaneously. ${ }^{1}$ This involves determining the baseline plasma cortisol level and then rapidly infusing a liter of normal saline containing $4 \mathrm{mg}$. of dexamethasone phosphate and 25 I.U. of corticotropin over an hour. The diagnosis is supported by improvement with infusion of saline and dexamethasone, the latter of which, unlike other steroids, will not interfere with plasma cortisol or urinary 17-OHCS. With Addison's disease, the corticotropin will not significantly raise the plasma cortisol level measured after completion of the infusion.

Some patients may need at least 5 liters of normal saline solution on the first day of treatment to maintain adequate intravascular volume. ${ }^{2}$ The acidosis and hyperkalemia usually require no specific therapy initially and are corrected by the fluid administration. One may opt to delay confirmatory diagnosis by testing adrenal reserve at a later date. ${ }^{1}$ In this instance, initial therapy should include a 200 - to $300-\mathrm{mg}$. bolus of hydrocortisone (Solu-Cortef), followed by an infusion of $100 \mathrm{mg}$. every 8 hours. Intramuscularly administered cortisone acetate (50 mg. every 12 hours) may also be given to ensure a continuous source of glucocorticoid in case the intravenous route is faulty, but this should not be the mainstay of therapy. With clinical progress, the dosage may be decreased 20-40 percent daily until the maintenance dose is achieved. Mineralocorticoid therapy is usually not needed until the daily dose of hydrocortisone reaches $75-100 \mathrm{mg}$. or its equivalent. Fludrocortisone acetate is the oral mineralocorticoid agent of choice.

Untreated Addison's disease may advance slowly, but all patients may rapidly deteriorate with adrenal crisis. Long-term survival depends on preventing and treating adrenal crisis. With treatment, the prognosis is very favorable.

\section{Report of case}

A 24-year-old Caucasian man was admitted on September 18, 1984, complaining of abdominal pain, nausea, vomiting, muscle cramps, and extreme weakness. Onset of the abdominal pain and cramps had been 7 days earlier; this had become progressively worse, with extreme nausea, vomiting, and inability to retain food or liquids coming on in the last 2 days. The patient admitted to feeling feverish on the first day, but he denied fever or chills after that. It was determined that he had noticed a decrease in his appetite, progressive weight loss, and fatigue throughout much of 1984 . He stated that his current weight was about 30 pounds less than it had been in December 1983. He also admitted to craving salt during the 3-month period prior to admission. He denied diarrhea.

His past medical history was most remarkable for idiopathic thrombocytopenic purpura, which had been diagnosed in 1978. Treatment had included splenectomy and vincristine, Imuran, and prednisone (up to $150 \mathrm{mg}$. per day), which had been tapered and then discontinued in 1980. Also in 1980, he had been diagnosed as having hypothyroidism secondary to Hashimoto's thyroiditis. Since then, except for the few days prior to admission, he had been taking $0.15 \mathrm{gm}$. of levothyroxine daily.

His social history was significant for separation from his wife in December 1983, and he blamed many of his physical problems, such as weight loss and "nervous stomach," on this. He denied any history of smoking or drug use and admitted only to minimal alcohol consumption. Other family history was noncontributory.

Initial physical examination revealed a thin, dehydrated man in moderate distress with abdominal discomfort. His height was 5'11', and he weighed 119 pounds. He was afebrile, his heart rate was 100 beats per minute, and his supine blood pressure was $82 / 40 \mathrm{~mm}$. Hg. Examination of the skin was remarkable for a bronze color. His nipples were dark, and there were several pigmented lesions over the right anterior chest, as well as small pigmented lesions on the right lower eyelid and the inside of the lower lip. The patient had dismissed the bronzing of his skin as a suntan; however, it was present in the pelvic area. Head, eyes, ears, nose, and throat examination was unremarkable except for dehydration of mucous membranes. The heart was regular without murmur and the lungs were clear. The abdomen was mildly tender in the periumbilical and suprapubic areas but was otherwise negative. Peripheral pulses were weak but palpable. Neurologic examination as well as the remainder of the physical examination was unremarkable.

Routine laboratory studies revealed the following values: leukocyte count, $12,200 / \mathrm{cu}$. mm., with a differential count of 39 percent segmented neutrophils, 48 percent lymphocytes, 8 percent eosinophils, and 5 percent monocytes; platelet count, 265,000/cu.mm.; hemoglobin, $16.2 \mathrm{gm} . / \mathrm{dl}$; and hematocrit, $44.8 \mathrm{ml} . / \mathrm{dl}$. Prothrombin and partial thromboplastin times were normal. Other values included: sodium, $131 \mathrm{mEq}$.L.; potassium, 7.2 mEq./L.; chloride, $97 \mathrm{mEq}$./L.; blood urea nitrogen, 52.6 mg./dl.; and creatinine, $2.8 \mathrm{mg} . / \mathrm{dl}$. Amylase and lipase 


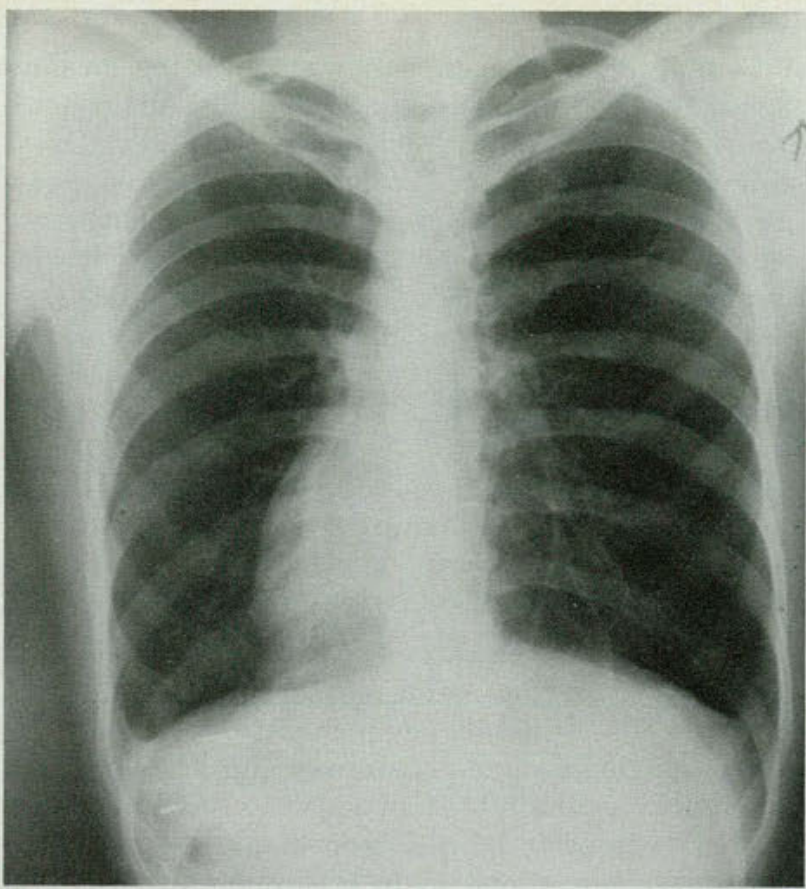

Fig. 1. Posteroanterior chest $x$-ray reveals decreased cardiac size, which is a common finding in Addison's disease.

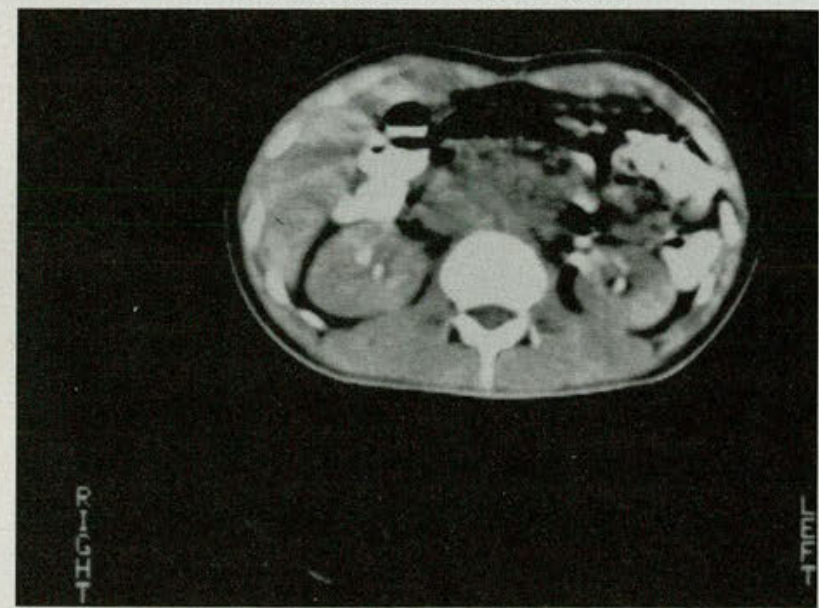

Fig. 2. Computed tomography scan of the abdomen reveals, in the area just below the head of the pancreas, an irregular, mixeddensity, periaortic mass $9.0 \mathrm{~cm}$. in diameter. Surgical exploration revealed this to be lymphadenopathy.

levels were normal. The serum glutamic-oxaloacetic level was slightly elevated to 73 I.U., and the total bilirubin value was normal at $0.94 \mathrm{mg}$./dl., with a direct component of $0.62 \mathrm{mg}$./dl. Arterial blood gas determinations revealed a $\mathrm{pH}$ of 7.477 , carbon dioxide partial pressure of $23.8 \mathrm{~mm}$. $\mathrm{Hg}$, oxygen partial pressure of 119.8 $\mathrm{mm} . \mathrm{Hg}$, and bicarbonate level of $17.8 \mathrm{mmol} . / \mathrm{L}$.

Chest roentgenography demonstrated a small cardiac silhouette (Fig. 1). Abdominal films showed scattered gas (perhaps suggestive of gastroenteritis). The admission electrocardiogram was normal.

Immediate treatment consisted of intravenous rehydration with normal saline solution with 5 percent dextrose at $200 \mathrm{cc} . / \mathrm{hr}$. This treatment alone corrected the prerenal azotemia and hyperkalemia after 36 hours, with lab parameters at that time as follows: potassium, $5.9 \mathrm{mEq} . / \mathrm{L}$.; blood urea nitrogen, $18.2 \mathrm{mg}$./dl.; creatinine, $1.3 \mathrm{mg} . / \mathrm{dl}$.; and sodium, $135 \mathrm{mEq} . \mathrm{L}$.

Meanwhile, other diagnostic tests were underway to document suspected Addison's disease. On the day after admission, the 8:00 a.m. and 4:00 p.m. plasma cortisol levels were both low, at $2.7 \mu \mathrm{g}$. $/ \mathrm{dl}$. and $2.1 \mu \mathrm{g}$./dl., respectively. The ACTH level was quite high at $1,500 \mathrm{pg} . / \mathrm{ml}$. (normal, $<82$ ). The thyroid profile, including thyroidstimulating hormone, was normal. The serum ceruloplasmin value was normal. Twenty-four urinary 17-ketosteroids (17-KS) and 17-hydroxycorticosteroids (17-OHCS) determinations were in the lower than normal range. Adrenal reserve was then tested by determining the a.m. plasma cortisol level, infusing the patient with $1,000 \mathrm{cc}$. of D5W with 40 units of ACTH at a rate of $125 \mathrm{cc} / \mathrm{hr}$., and then redetermining an 8-hour plasma cortisol level, as well as 24-hour 17-KS and 17-OHCS values. The low results indicated limited adrenal reserve.

Abdominal computed tomography (CT) scanning revealed a $9.0-\mathrm{cm}$. periaortic mass just below the pancreas, which was reported to most likely be lymphomatous in origin. Multiple lymph node involvement was noted descending the periaortic lymphatic chain (Fig. 2).

With a confirmed diagnosis of Addison's disease, the patient was placed on a regimen of cortisone acetate (100 $\mathrm{mg}$./day in divided doses) and fludrocortisone acetate $(0.2$ $\mathrm{mg}$./day), the latter for mineralocorticoid replacement. On the seventh hospital day, the patient's status had improved to the point where he was on a general diet, and intravenous fluids were discontinued. However, noninvasive work up of the abdominal mass continued; this included chest and pelvis CT scanning, as well as CEA and tuberculin skin testing, all with negative results.

A decision was made for surgical abdominal exploration and biopsy for possible malignancy, and, on the ninth hospital day, the patient underwent laparotomy with retroperitoneal dissection. Excision of the lymphoid-like growth on the superior portion of the pancreas, near the head, was performed. An additional lymphoid-like mass was excised near the common bile duct. The left adrenal gland was identified and wedge biopsied. Needle biopsy of the liver also was performed.

The biopsy specimens did not reveal malignancy. The lymphadenopathy was reported to be of unexplained etiology. However, it was noted that the specimens had characteristics that could be explained by autoimmune phenomenon or viremia, and also that were not inconsistent with sarcoidosis or amyloidosis.

The patient was supported with increased corticosteroid dosages in the perioperative period and made progressive postoperative recovery. By the sixth postoperative day, he had been tapered to maintenance dosages of cortisone acetate $(25 \mathrm{mg}$. in the morning and $12.5 \mathrm{mg}$. with dinner) and Florinef ( $0.1 \mathrm{mg}$. daily). He had also been maintained on Synthroid $(0.15 \mathrm{mg}$. daily) throughout his hospitalization. He was also supported with nutritional parenteral supplements.

The patient was discharged on October 5, 1984, with a 
final diagnosis as follows: (1) newly found Addison's disease; (2) probable acute viral gastroenteritis; (3) abdominal lymphadenopathy, which probably was secondary to nos. 1 and 2; (4) treated Hashimoto's thyroiditis; and (5) prerenal azotemia and electrolyte imbalance secondary to nos. 1 and 2, which was corrected. His treatment on discharge and to this day consists of cortisone acetate, Florinef, and Synthroid in the maintenance doses mentioned previously. He has been seen on several follow-up visits, and, gaining weight with increasing appetite. Unfortunately, after several visits, he was lost to follow-up.

\section{Discussion}

Primary adrenocortical insufficiency is relatively rare; the estimated incidence in Western countries is around 50 per million population. ${ }^{3}$ Schmidt's syndrome (idiopathic hypothyroidism and Addison's disease) was once thought to be extremely rare, but it now is recognized to be a segment of polyendocrinic autoimmune disease. ${ }^{4,5}$ A number of endocrinic and other diseases that presumably are caused by autoimmune pathogenic mechanisms have been shown to occur with increased frequency in the same individual or members of the same family. ${ }^{6}$ These include chronic lymphocytic (Hashimoto's) thyroiditis, Graves' disease, Addison's disease, hypoparathyroidism, hypopituitarism, primary gonodal failure, autoimmune oophoritis and orchitis, insulin-dependent diabetes mellitus, myasthenia gravis, idiopathic thrombocytopenic purpura, pernicious anemia, alopecia, Sjogren's syndrome, systemic lupus erythematosus, and scleroderma. The incidence of malignancy also seems to be increased in the affected families.

Recently, it has been recognized that some of these diseases occur in individuals who possess specific HLA antigens. It has been proposed that HLA typing can become an important diagnostic tool in identifying individuals at risk for autoimmune disease. The diseases in the case presentedAddison's disease, Hashimoto's thyroiditis, and idiopathic thrombocytopenic purpura-have all been demonstrated to be quite consistently associated with HLA-B8 antigens. ${ }^{6}$

In the case presented, the differential diagnosis initially included secondary adrenal insufficiency, either hypopituitarism secondary to pituitary disease or, especially with the patient's past history of exogenous steroid therapy, suppression of the hy- pothalamic pituitary axis. ${ }^{7,8}$ However, the extremely high ACTH value indicated primary adrenal insufficiency.

After the acute phase of therapy, patient education assumes an important role. Many adrenal crises are preventable. Patients should carry medical identification and be knowledgeable concerning the symptoms of worsening adrenal insufficiency. Family and friends should also be aware of these symptoms and their importance. Minor surgery, infection, and other stress is best managed by doubling the daily steroid dose for a few days until the stressful event has ended.

\section{Summary}

The patient in the case reported had Addison's disease, Hashimoto's thyroiditis, and idiopathic thrombocytopenic purpura. When an autoimmune disease is diagnosed, the clinician must be suspicious for related autoimmune disorders.

\footnotetext{
1. Liddle, G.W.: The adrenals. In Textbook of endocrinology, edited by R.H. Williams. Ed. 6. W.B. Saunders Co., Philadelphia, 1981

2. Hamburger, S., and Rush, D.R.: Adrenal crises. Top Emerg Med 4:75-8, Jul 83

3. Tyrrell, J.B., and Baxter, J.D.: Disorders of the adrenal cortex. In Cecil Textbook of medicine, edited by J.B. Wyngaarden and L.H. Smith. W.B. Saunders Co., Philadelphia, 1985

4. Appel, G.B., and Holub, D.A.: The syndrome of multiple endocrine gland insufficiency. Am J Med 61:129-33, Jul 76

5. Loeb, J.N., Polyglandular disorders. In Cecil textbook of medicine, edited by J.B. Wyngaarden and L.H. Smith. W.B. Saunders Co., Philadelphia, 1985

6. Valenta, L.J., et al.: Correlation of the HLA-A1, B8 haplotypes with circulating autoantibodies in a family with increased incidence of autoimmune disease. Acta Endocrinolog 100:143-9, May 82

7. Harrington, W.J., et al: Treatment of idiopathic thrombocytopenic purpura. Hosp Prac 18:205-10,215,218-20, Sep 83

8. Zurbrugg, R.P., Zimmermann, E., and Oetliker, O.H.: Adrenocortical function following long-term corticosteroid treatment, Helv Paediatr Acta 29:221-35, Nov 74
}

Accepted for publication in August 1986. Updating, as necessary, has been done by the authors.

At the time this paper was written, Dr. Meeker was an intern at Davenport Medical Center (DMC), Davenport, Iowa. He is now a resident in family practice at St. Luke's Hospital, Milwaukee, Wisconsin. Dr. King is an attending physician in internal medicine at DMC and Dr. Arnold is an attending general surgeon at DMC.

Dr. Meeker, 3131 South 28th Street, Milwaukee, Wisconsin 53215 . 


\section{A defense against cancer can be cooked up in your kitchen.}

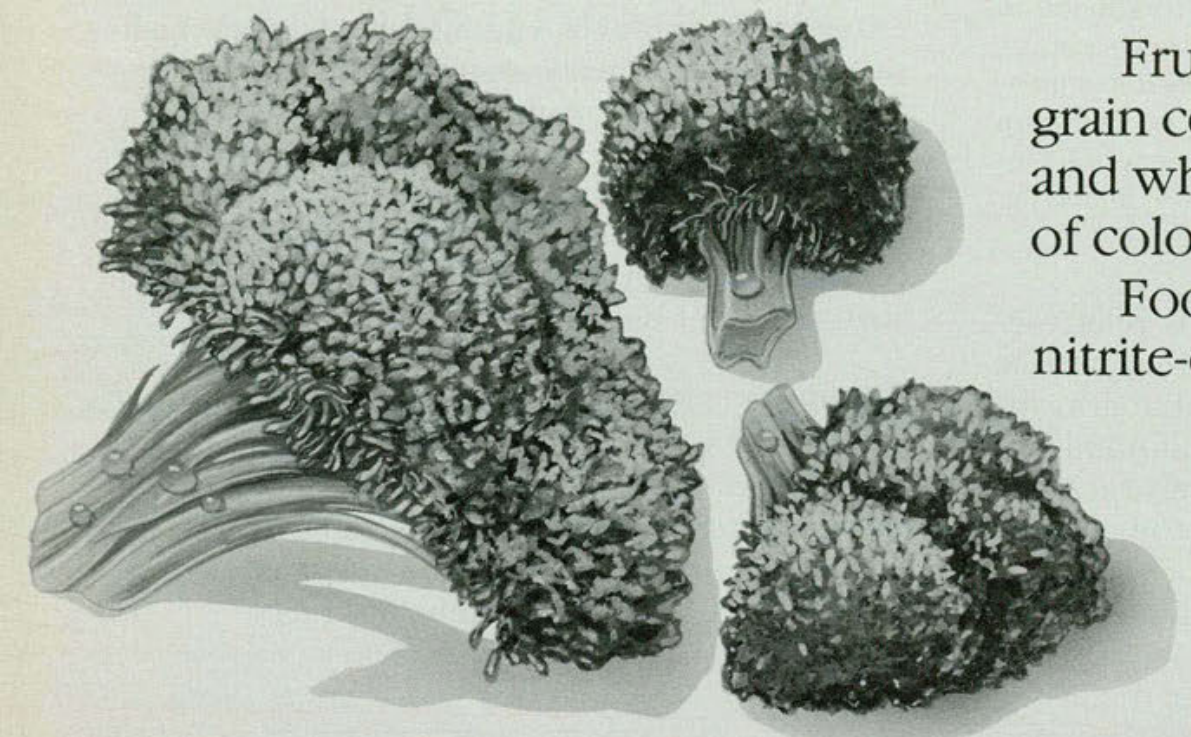

Fruits, vegetables, and wholegrain cereals such as oatmeal, brar and wheat may help lower the ris of colorectal cancer.

Foods high in fats, salt- or nitrite-cured foods like ham, and

There is evidence that diet and cancer are related. Some foods may promote cancer, while others may protect you from it.

Foods related to lowering the risk of cancer of the larynx and esophagus all have high amounts of carotene, a form of Vitamin A which is in cantaloupes, peaches, broccoli, spinach, all dark green leafy vegetables, sweet potatoes, carrots, pumpkin, winter squash and tomatoes, citrus fruits and brussels sprouts.

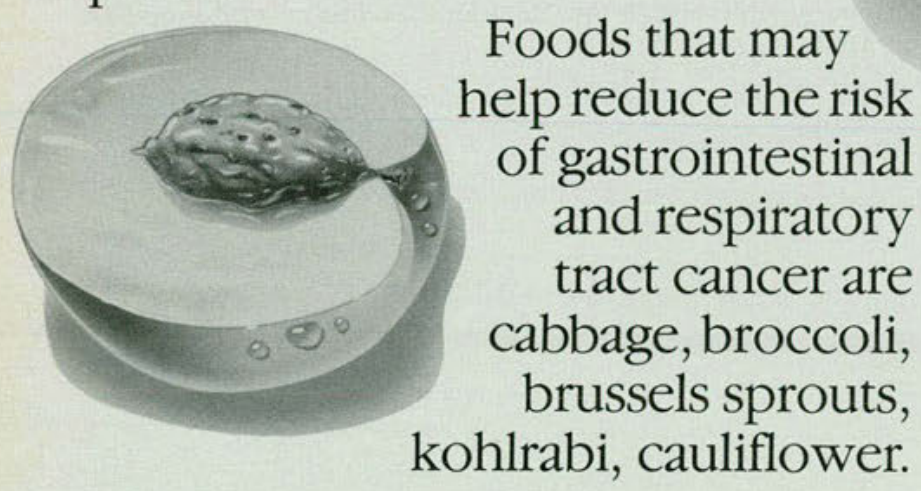

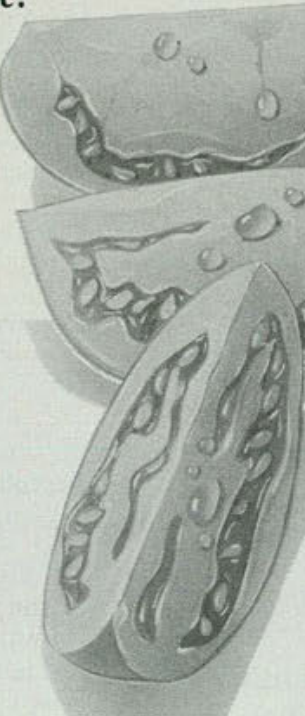

fish and

types of sausages smoked by trad tional methods should b eaten in moderation.

Be moderate in consumption of alcc hol also.

A good rule of thumb is cut down $\mathrm{O}$ fat and don't be fat.

Weight reduction may lower cancer risk. Our 12- year study of nearly a million Americans uncovere high cancer risks particularly among people $40 \%$ or more overweight.

Now, more than ever, we know you can cook up your own defense against cancer. So eat healthy and be healthy.

No one faces cancer alone.

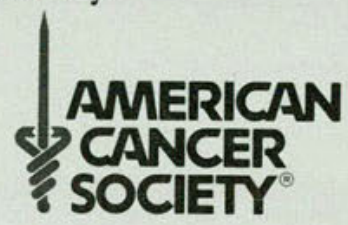

